\title{
Un Acercamiento a datos abiertos en salud y su estado actual en Colombia
}

\author{
An Approach to Open Data in Health and its Current Status in Colombia \\ Uma aproximação aos Dados Abertos em Saúde e seu atual estado na Colômbia
}

DOI: http://dx.doi.org/10.21803\%2Fpenamer.11.21.528

\author{
Walter Hugo Arboleda Mazo \\ https://orcid.org/0000-0003-4937-5359 \\ Raquel Anaya Hernández \\ https://orcid.org/0000-0002-9187-7427
}

\section{¿Cómo citar este artículo?}

Arboleda, W. \& Anaya, R. (2018). Un Acercamiento a datos abiertos en salud y su estado actual en Colombia. Pensamiento Americano, 11(21), 110-126 110

\section{Resumen}

El uso de datos abiertos es una estrategia que promueve la transparencia y visibilidad de la gestión de entidades gubernamentales con el propósito de proveer datos oportunos y fiables a los diferentes sectores para la toma de decisiones informada. Países como Estados Unidos y el Reino Unido han definido marcos de referencia que definen una estrategia unificada para datos abiertos en salud, denominada Open Health Data (OHD); asimismo la Organización Mundial de la Salud (OMS) propone una red denominada Health Metrics Network (HMN), para apoyar a los países en su propósito de mejorar la salud global a través del fortalecimiento de los sistemas de información de la salud. En Colombia la estrategia de datos abiertos para apoyar las iniciativas de gobierno en línea, ha venido fortaleciéndose y madurando. Sin embargo, aún existen retos abiertos en el fortalecimiento de datos abiertos en el tema de salud que deben ser enfrentados, como: el cambio de cultura de los organismos responsables de los datos para compartirlos, donde la formación de los ciudadanos es clave; el empoderamiento de las entidades territoriales que les permita entender y visualizar las potencialidades de este enfoque, la puesta en marcha de los lineamientos acerca de la anonimización de los datos individuales para satisfaces las reglamentaciones en términos de privacidad de información y la estimulación en aprovechamiento de los datos abiertos por parte de profesionales de las TIC para la creación de nuevos productos y servicios, garantizándose un verdadero impacto de los datos abiertos gubernamentales en el país. Se hace una propuesta de un modelo operacional en el que se ilustra la manera como todos los actores y sistemas de información se puede articular en un ecosistema de sistemas de información en salud que se integra con el catálogo de datos abierto.

PALABRAS CLAVES: Datos abiertos, Datos abiertos en salud, Catalogo de datos abierto, Gobierno en Línea, Plan Decenal de Salud, Anonimización de Datos.

\begin{abstract}
The use of open data is a strategy that promotes transparency and visibility of the government entities management in order to providing timely and reliable data to the different sectors for informed decision making. Countries like the United States and the United Kingdom have defined frameworks that define a unified strategy for open data on health, called Open Health Data (OHD); also the World Health Organization (WHO) proposes a network called Health Metrics Network (HMN), to support countries in their purpose of improving global health through the strengthening of health information systems. In Colombia, the open data strategy which aims to support online government initiatives, has been strengthening and maturing. However, there are still ongoing challenges that must be faced in strengthening health open data, such as: culture change of the organisms responsible for the data to share, where the key factor is citizenship training; territorial entities empowerment
\end{abstract}


that allows them to understand and visualize this approach's potential, guidelines implementation on the individual data anonymization to satisfy the regulations in terms of information privacy and the stimulation of the use of open data by ICT professionals for new products and services creation, guaranteeing a true government open data impact in the country. A proposal is made for an operational model that illustrates the way in which all actors and information systems can be articulated in a health information systems ecosystem that is integrated with the open data catalog.

KEYWORDS: Open data, Health open data, Open data catalog, Government Online, Ten-Year Health Plan, Data Anonymization

\section{Resumo}

O uso de dados abertos é uma estratégia que promove a transparência e visibilidade da gestão de entidades governamentais com o propósito de prover dados oportunos e confiáveis aos diferentes setores para tomar decisões informadas. Países como os Estados Unidos e o Reino Unido definiram marcos de referência que definem uma estratégia unificada para os dados abertos em saúde, denominada Open Health Data (OHD); mesmo assim, a Organização Mundial de Saúde (OMS) propõe uma rede denominada Health Metrics Netword (HMN), para apoiar aos países em seu propósito de melhorar a saúde global através do fortalecimento dos sistemas de informação da saúde. Na Colômbia a estratégia de dados abertos para apoiar as iniciativas de governo em rede se fortalece. Todavía, ainda existem desafios abertos no fortalecimento dos dados abertos no tema da saúde que devem ser enfrentados, como: a mudança de cultura dos organismos responsáveis dos dados para compartilhá-los, onde a formação dos cidadãos é fundamental; o empoderamento das entidades territoriais que os permita entender e visualizar as potencialidades deste enfoque, o andamento dos lineamentos sobre a anonimização dos dados individuais para satisfazer os regramentos em termos de privacidade da informação e estímulo em aproveitamento dos dados abertos por parte dos profissionais das TIC para a criação de novos produtos e serviços, garantindo um verdadeiro impacto dos dados abertos governamentais em seu país. Efetua-se uma proposta de modelo operacional o qual se ilustra a maneira como todos os atores e sistemas de informação podem ser articulados em um ecossistema de sistemas de informação em saúde que se integra com o catálogo de dados abertos.

PALAVRAS CHAVE: Dados abertos, Dados abertos em saúde, Catálogo de dados aberto, Governo em redes, Plano Decenal de Saúde, Anonimização de Dados.

\section{Perfil}

Especialista en redes corporativas e integración de la universidad de San Buenaventura; Magister en Ingeniería, Universidad EAFIT. Docente de la Facultad de Ingeniería, Corporación Universitaria Adventista. warboleda@unac.edu.co

\section{Perfil}

Doctorado en Informatica, Universidad Politecnica de Valencia Espana. Investigadora Facultad de Ingenieria, Corporacion Universitaria Adventista.

raquel.anaya.hdez@gmail.com
Walter Hugo Arboleda Mazo Ingeniero de sistemas de la universidad de San Buenaventura.

\section{Raquel Anaya Hernández}

Ingeniera de sistemas de la universidad industrial de Santander. 


\section{Introducción}

L

a evolución de la tecnología de información y comunicaciones (TIC) ha propiciado que los gobiernos, el sector productivo y el sector académico, reconozcan la importancia de las TIC como mecanismo que permite disponer de datos precisos, correctos, oportunos y estructurados, de tal manera que propicie un proceso de toma de decisiones informada e incluso favorezca la gobernabilidad y continuidad de las acciones tanto del sector público como del sector privado, línea conocida como datos abiertos. Hoy en día el uso de datos abiertos es una estrategia reconocida a nivel mundial que busca la transparencia y efectividad de los gobiernos (CDC, 2018)(NCCDPHP, 2018).

Los datos abiertos ha propiciado avances tecnológicos en estandarización y manipulación masiva de los datos y en la definición de políticas y referentes que evaIúan la efectividad de este enfoque en un gobierno (SOCRATA, 2018), ha generado posibilidades de nuevas líneas de negocio (CDC, 2018) (CDPH, 2018), ha promovido una conciencia general de la importancia de éstos como activos de un gobierno para manejo de datos como la ecología (DATA. NJ.GOV, 2018) y el sector agrario (OPEN. CANADA.CA, 2018)y ha generado nuevas líneas de investigación que van más allá de los aspectos técnicos.

De otra parte, también se perciben retos abiertos desde la perspectiva política y social, y se proponen indicadores que analizan el impacto de dichos datos como medidores de impacto real de transparencia de los gobiernos (DATA.GOV, 2018); hacia el futuro próximo se espera que los datos abiertos propicie unas relaciones entre los gobiernos y los ciudadanos orientados hacia la negoción de las tensiones éticas y económicas que existen entre el gobierno y los diferentes actores involucrados (ODC, 2018).

El objetivo de este trabajo es hacer un acercamiento de los datos abiertos desde sus orígenes, y su evolución a nivel mundial y una reflexión sobre el estado actual a nivel de Colombia. Para efectos prácticos se tomará la temática de salud, como punto de análisis, considerando que este en un tema de interés central a nivel mundial. En la segunda sección se presentan las características de los datos abiertos, la evolución que ha tenido el concepto, los movimientos e iniciativas de cooperación que se han generado alrededor del tema y el estado actual de datos abiertos en salud a nivel internacional. En la tercera sección se describe la evolución en datos abiertos y el estado actual de la estrategia datos abiertos en salud en Colombia y se describe las diferentes formas de participación en que las diversas organizaciones. En la cuarta sección, se describen los retos abiertos en adopción del enfoque en el tema de la salud, a la luz de referentes analizados a nivel mundial. Finalmente, en la sección sexta se presentan las conclusiones.

\section{Entendiendo los datos abiertos}

De acuerdo al Open Data Charter, los datos abiertos son "datos digitales que son puestos a disposición con las características técnicas y jurídicas necesarias para que puedan ser usados, reutilizados y redistribuidos libremente por cualquier persona, en cualquier momento y en cualquier lugar"1.

De esta definición se derivan dos aspectos importantes de los datos abiertos: de una parte, las características técnicas están referidas al almacenamiento de datos en archi-

1 opendatacharter.net

Pensamiento Americano Vol. 11 - No. 21 · Julio-Diciembre • Corporación Universitaria Americana • Barranquilla, Colombia · ISSN: 2027-2448 . 
vos electrónicos que deben cumplir unos formatos de descripción e intercambio ya establecidos por comunidades internacionales $\mathrm{y}$, de otra parte, las características jurídicas que hacen referencia al uso de la información verídica y características de publicación de información no sensible y que sea respetuosa con el derecho de privacidad de la información. Los datos abiertos están directamente asociados a las políticas de gestión de un gobierno y por lo tanto representa un indicador sensible que muestra la transparencia y gobernabilidad de un país, buscando empoderar a los sectores públicos, privados y comunidad en general, para que se tomen decisiones informadas.

En esta sección se analizarán las características de los datos abiertos, la evolución y movimientos que se han generado alrededor de este tema.

\subsection{Características de los datos abiertos}

Para que los datos generados por diferentes entidades gubernamentales se consideren datos abiertos con las cualidades esperadas, debe realizarse sobre un ellos un tratamiento cuidadoso que sigue los siguientes pasos generales: se identifican los datos almacenados en los sistemas transaccionales que van a ser candidatos a datos abiertos; el sistema que gestiona el catálogo de datos abiertos recibe datos gubernamentales seleccionados, los cataloga conservando aspectos de calidad de cinco estrellas, luego los limpia y los convierte a un formato que sea leíble por la plataforma y los publica cumpliendo con el estándar definido; los datos quedan disponibles para que puedan ser bajados directamente o para que se sean consumidos por aplicaciones informáticas. Buena parte de estos procesos se realizan de forma automática y computarizada y con una frecuencia establecida para garantizar que los datos se encuentren actualizados (ODC, 2018).

\section{3 opendatacharter.net}

En el año 2007 fueron formulados, por Open Government en los Estados Unidos, los ocho principios de los datos gubernamentales los cuales fueron mejorados por la Sunlight Foundation en el año mismo año, creándose diez principios ${ }^{2}$ que proponen características que deben cumplir los datos para que sean abiertos: los datos deben ser completos, primarios, oportunos, accesibles, producibles por maquinas, su acceso no debe ser discriminatorio, no deben ser en formatos propietarios, deben ser publicados bajo licencia libre de acceso, deben permanecer en el tiempo y no deben tener costo de uso.

Estos principios garantizan el acceso a cualquier persona o computadora, en cualquier momento, conservándose recomendaciones y convenciones de organizaciones como: W3C, BLSG en Australia y SAGA en Alemania. Todos los conjuntos de datos gubernamentales abiertos deben ser publicados bajo licencia Creative Commons Attribution 3.0; los datos deben ser auto contenidos, lo que significa que los mismos datos deben describir cuál es su contenido, lo que se conoce como metadatos; debe garantizarse un control de versiones adecuado y un archivado permanente; además deben cumplir con su principal característica de ser libres de usar, diseminar y reusar, sin ningún costo (Eibl, 2013).

\subsection{Movimientos alrededor de datos abiertos}

En el año 2009 los gobiernos de Estados Unidos y Reino Unido realizaron acuerdos para apoyar el establecimiento de los datos abiertos gubernamentales, en Estados Unidos se lanzó el portal, en mayo de $2009^{3}$, y en Reino Unido en enero de $2010^{4}$. A partir de allí han

2 https://sunlightfoundation.com/policy/documents/ten-open-data-principles/ 
aparecido varios movimientos proactivos apoyando también esta directiva la cual busca el reúso de la información como un bien público, la aparición de nuevas oportunidades de negocio, la mejora de la transparencia y la información oportuna para los ciu- dadanos. La European Commission abordo los datos gubernamentales abiertos desde el año 2003, mediante una directiva del sector público y en el año 2011 definió el Open Data Package. Es así como en enero de 2012, ya había más de 700.000 conjuntos de datos

Tabla 1. Seis principios de los datos abiertos según ODC

\begin{tabular}{|c|c|}
\hline Principio & Implicaciones \\
\hline $\begin{array}{l}\text { Los datos deben ser } \\
\text { abiertos }\end{array}$ & $\begin{array}{l}\text { Desarrollar y adoptar políticas y prácticas para asegurar que los datos generados por el } \\
\text { gobierno son abiertos por defecto, generando una clara justificación en caso de que éstos } \\
\text { no sean liberados. Establecer una cultura en la sociedad civil y el sector privado para que } \\
\text { sean conscientes de las responsabilidades y beneficios de datos abiertos. Observar leyes y } \\
\text { estándares con respecto a seguridad, privacidad, confidencialidad y propiedad intelectual. }\end{array}$ \\
\hline $\begin{array}{l}\text { Los datos deben ser } \\
\text { oportunos y com- } \\
\text { prensivos }\end{array}$ & $\begin{array}{l}\text { Liberar los datos abiertos de alta calidad en forma oportuna, sin demora indebida; publica- } \\
\text { dos en la forma original de su generación; desagregados a los niveles más bajos de adminis- } \\
\text { tración, como sexo, edad, ingresos y otras categorías; que permitan retroalimentación conti- } \\
\text { nua y revisiones para asegurar la calidad; que se apliquen prácticas consistentes de gestión } \\
\text { de la información del ciclo de vida y se aseguren copias de datos históricos que conserven } \\
\text { el valor; que se haga transparente la información acerca de los procesos utilizado para la } \\
\text { recolección y publicación de los datos. }\end{array}$ \\
\hline $\begin{array}{l}\text { Los datos deben ser } \\
\text { accesibles y utiliza- } \\
\text { bles. }\end{array}$ & $\begin{array}{l}\text { Deben ser publicados en un portal central, de manera que sean fácilmente visibles y dispo- } \\
\text { nibles; deben ser distribuidos en diferentes formatos abiertos, para que puedan ser procesa- } \\
\text { dos por computadora y utilizados por personas; que puedan ser accesibles por una amplia } \\
\text { gama de usuarios; que su acceso sea de forma gratuita, bajo una licencia abierta y que } \\
\text { puedan ser accedidos sin un registro obligatorio. }\end{array}$ \\
\hline $\begin{array}{l}\text { Los datos deben ser } \\
\text { comparables e inte- } \\
\text { roperables. }\end{array}$ & $\begin{array}{l}\text { Deben ser compartidos en formatos estándares; deben incluir metadatos núcleo que están } \\
\text { disponibles en formatos legibles para personas y máquinas; descritos completamente en } \\
\text { un lenguaje claro y sencillo y que tengan suficiente información para comprender el origen } \\
\text { fortalezas, debilidades y limitaciones de análisis de los datos; comprometerse con iniciativas } \\
\text { que fomentan el aumento de la interoperabilidad. }\end{array}$ \\
\hline $\begin{array}{l}\text { Orientados a mejorar } \\
\text { la gobernabilidad y } \\
\text { el involucramiento } \\
\text { de los ciudadanos }\end{array}$ & $\begin{array}{l}\text { Asegurar que la información publicada como resultado de leyes contra la corrupción o trans- } \\
\text { parencia se libera en forma de datos abierta; proporcionar programas de capacitación para } \\
\text { asegurar que los empleados del gobierno son capaces de utilizar los datos abiertos para el } \\
\text { desarrollo de políticas; debe existir compromiso con la libertad de información / Acceso a la } \\
\text { Información / Derecho de la comunidad a la disponibilidad de datos abiertos solicitados; par- } \\
\text { ticipación activamente con líderes de la comunidad, sociedad civil y el sector privado para } \\
\text { determinar qué datos se requieren para el rendimientos de cuentas del gobierno; respetar } \\
\text { el derecho de los ciudadanos a la libertad de expresión permitiendo el uso de datos abiertos } \\
\text { para identificar la corrupción o criticar a los gobiernos; promover el uso de datos abiertos } \\
\text { para desarrollar soluciones innovadoras, basadas en la evidencia de políticas que benefician } \\
\text { a todos los miembros de la sociedad, incluyendo el empoderamiento de las comunidades } \\
\text { marginadas. }\end{array}$ \\
\hline $\begin{array}{l}\text { Los datos deben pro- } \\
\text { mover la innovación }\end{array}$ & $\begin{array}{l}\text { Motivar a ciudadanos, sociedad civil y organismos del sector privado e instituciones multila- } \\
\text { terales para abrir archivo de datos creados y administrados por éstas con el fin de avanzar } \\
\text { hacia un ecosistema más rico de datos abierto procedentes de múltiples fuentes; crear o ex- } \\
\text { plorar posibles asociaciones que apoyen la liberación de los datos abiertos con el propósito } \\
\text { de maximizar el impacto de los datos reales a través de su uso; crear o apoyar programas e } \\
\text { iniciativas que favorezcan el desarrollo de co- creación de conjuntos de datos de oro, visuali- } \\
\text { zaciones, aplicaciones y otras herramientas basadas en datos abierta; comprometerse con } \\
\text { las escuelas e instituciones de educación post-secundaria para incrementar la investigación } \\
\text { en datos abiertos aumentó incorporación de datos en los programas de enseñanza de alfa- } \\
\text { betización; }\end{array}$ \\
\hline
\end{tabular}

3 http://www.data.gov

4 http://data.gov.uk 
gubernamentales abiertos de toda Europa cargados en el sitio de este proyecto (TWC LOGD Linking Open Government Data) ${ }^{5}$.

Actualmente los esfuerzos internacionales de datos abiertos confluyen en una iniciativa central denominada Carta de Datos Abiertos $(\text { ODC })^{6}$, creada en el 2015, como una colaboración entre el sector gubernamental y grupos de expertos, conformado por 17 países alrededor del mundo y 34 gobiernos regionales, con el propósito de definir conjuntamente los principios y lineamientos de la manera como los diferentes gobiernos deben publicar información.

La ODC definió seis principios de datos abiertos que se encuentran resumidos en la tabla 1. Se puede observar que los principios establecidos por la ODC, están orientados hacia aspectos estratégicos que direccionan el uso y aprovechamiento de los datos abiertos, a diferencia de la propuesta inicial de principios que aborda los aspectos operativos. Existe un indicador mundial que clasifica a los países con base en el nivel de publicación de datos gubernamentales claves, beneficios y pruebas de su impacto, denominado Open Data Barometer (ODB) ${ }^{7}$, que en el reporte del 2016 está encabezado por Inglaterra, Canadá, Francia y Estados Unidos. Los gobiernos de los países de Latinoamericanos ya están empezando a entender la potencialidad de este enfoque como una estrategia que apoya la transparencia y gobernabilidad. México, Uruguay y Brasil, por ejemplo, se encuentran entre los 20 primeros puestos del reporte ODB de 2016, ocupando los puestos 11, 17, 18, respectivamente. Colombia empieza a tener una tendencia de mejora figurando en el puesto 24 , superan- do a Chile que se encuentra en el puesto 26.

A nivel latinoamericano se destaca la red de organizaciones de la región denominada Iniciativa Latinoamericana de Datos Abiertos (ILDA) ${ }^{8}$ la cual busca impulsar la investigación, apropiación y uso de los datos abiertos en la región. Ya se han empezado a mostrar resultados interesantes de esfuerzos conjuntos para realizar estudios de datos abiertos en diversos temas como educación (Khelladi, 2015) , rama judicial (Elena, 2015), ciudades abiertas e innovadoras (Bonina, 2015) y en el estudio del dengue (Pane, Ojeda, \& Valdez, 2015).

\subsection{Estado actual de datos abiertos para la salud a nivel internacional}

Los dos países que han tenido el liderazgo de datos abiertos en salud, a nivel mundial, son Estados Unidos e Inglaterra. El Departamento de Salud y Servicios Humanos de Estados Unidos (HHS) ${ }^{9}$ tiene un Plan de Gobierno Abierto el cual ha sido actualizado y creado desde antes del año 2012; en su plan actual para 2014-2018 tiene como meta aumentar la transparencia de servicios de salud en costos y opciones, permitiendo usar los datos de millones de ciudadanos que están en el sistema de salud. El Centro de Control y Prevención de Enfermedades (CDC) ${ }^{10}$ genera datos abiertos, que pueden ser accedidos y exportados para diversos usos; se tiene disponible además el Catálogo de Indicadores en Salud $(H I W)^{11}$ el cual provee servicios web para el acceso a grandes conjuntos de datos, ofreciendo mejor calidad en los datos abiertos federales. En el proyecto de manejo de datos abiertos en salud se encuentran involucradas entidades nacionales como la Oficina de Prevención y Promoción Nacional y el Consejo de Salud Pública, los cuales promueven estilos de

http://www.healthindicators.gov/ 
vida saludable bajo directivas como: vida libre de tabaco, prevención en abuso de las drogas y uso excesivo del alcohol, comer sano, no sedentarismo, maltrato y violencia, salud sexual y reproductiva, bienestar mental y emocional (National Prevention Council, 2011).

En Inglaterra, los Datos Abiertos en Salud se encuentra disponibles a través del portal oficial $^{12}$, el cual articula datos abiertos en las diferentes temáticas de interés del gobierno, incluido el tema de la salud. Por su parte, el Health \& Social Care Information Centre ${ }^{13}$ ofrece datos abiertos a proveedores, investigadores y pacientes. En enero de 2014 el HHS en los Estados unidos y el NHS en Inglaterra firmaron un acuerdo bilateral para compartir información, herramientas y servicios, desde la su firma la Iniciativa de Datos en Salud ha compartido cerca de 1.000 conjuntos de datos de abiertos (Noveck, 2014).

Por su parte, la Organización Mundial de la Salud lanzó en el 2005 una red, denominada Health Metrics Network (HMN), que buscar apoyar a los países en su propósito de mejorar la salud global, fortaleciendo los sistemas de información de la salud, de tal manera que la información que éstos generan sea soporte para toma de decisiones sustentadas en los datos como evidencia. En el año 2011, dicha red definió un marco de trabajo y estándares para los sistemas de información, denominado HMN Framework, con el propósito de que fuera mundialmente aceptado, útil para la recolección, reporte y uso de la información de salud de todos los países.

El HMN Framework define 6 componentes de un sistema de información de la salud y precisa la manera como éstos interactúa entre sí. Los componentes se agrupan a su vez, en tres categorías: entradas (recursos), proce- sos (indicadores, fuentes de datos, gestión de los datos) y salidas (producción diseminación y uso de información). Un elemento importante de este framework son los indicadores del área de la salud, los cuales se clasifican en 3 grupos: (1) Indicadores determinantes de salud, que incluyen aspectos socio económicos, ambientales, demográficos y genéticos; (2) indicadores del sistema de salud mismo, tales como políticas, organización, recursos humanos y financieros, infraestructura de salud, equipos y materiales, recursos humanos; también comprende indicadores acerca de la disponibilidad y calidad tanto de los servicios como del sistema de información; (3) Indicadores que evalúan la efectividad del sistema de salud como el nivel de mortalidad, morbilidad, discapacidad y bienestar.

El HMN Framework hace énfasis en la aplicación de políticas y procesos que garanticen la calidad de los datos, teniendo en cuenta la frecuencia de su recolección, el tiempo transcurrido entre la recolección y su publicación, la consistencia de los datos, y la representatividad, desagregación y confidencialidad de los mismos. Propone procesos para la extracción e integración de datos de diferentes fuentes con el propósito de crear un repositorio integrado de datos, que faciliten su consulta y manipulación a diferentes niveles de detalle, por diferentes actores del sistema.

En los países de Latinoamérica las iniciativas de datos abiertos en salud son aun incipientes: las entidades de salud de los paises empiezan a publicar algunos datos que son de interés ciudadano como la información de prestadores de servicios de salud y tasa de enfermedades transmisibles (Uruguay) ${ }^{14}$; listado de farmacias e información acerca de sus turnos (Chile) ${ }^{15}$; informes estadísticos por grupo de hospitales (Perú) ${ }^{16}$, datos de auto-

$12 \quad$ data.gov.uk

13 hscic.gov.uk

Pensamiento Americano Vol. 11 - No. 21 • Julio-Diciembre · Corporación Universitaria Americana • Barranquilla, Colombia • ISSN: 2027-2448 . http://coruniamericana.edu.co/publicaciones/ojs/index.php/pensamientoamericano 
cuidado, indicadores de salud, procedimientos y medicamentos del POS, indicadores de salud de EPS e IPS y aplicaciones de apoyo al cuidado de la salud (Colombia) ${ }^{17}$.

\section{Panorámica de datos abiertos en Colombia}

\subsection{Estado Actual de datos abiertos en Colombia}

El tema de datos abiertos en Colombia ha sido liderado por el Ministerio de las Tecnologías de Información y Comunicaciones (MINTIC), como organismo responsable de definir las acciones para liderar la política de gobierno en línea. El artículo 230 del Plan Nacional de Desarrollo 2010 - 2014, presentó el programa "Gobierno en línea como estrategia de un buen gobierno", en la cual todas las entidades de administración pública debían adelantar acciones de acuerdo a los lineamientos establecidos por el MINTIC ${ }^{18}$. Con la evolución de la Estrategia de Gobierno en Línea en el 2011 se incorporan nuevas temáticas como Gobierno Abierto y los Datos Abiertos y surge la propuesta de Modelo de Datos Abierto propuesta por el MINTIC, la cual está estructurada en capas, para identificar el papel que los diferentes actores involucrados (gobiernos, entidades territoriales, proveedores y clientes de los datos, personal técnico de apoyo) cumplen en una iniciativa de datos abiertos.

En el año 2012, se iniciaron en Colombia las primeras acciones para definir un Catálogo de Datos Abiertos ${ }^{19}$ producto de un proyecto piloto, estimulando el desarrollo de servicios basados en datos públicos; dicho catálogo estaba basado en el Modelo de Datos Abiertos definido por el MINTIC en un portal desarrollado en .NET el cual contenía las colecciones provenientes de las entidades nacionales y territoriales, debidamente registradas con su usuario y contraseña, las cuales cargaban sus datos al catálogo. En la actualidad el catálogo de datos abiertos funciona usando Socrata 20 , catálogo de datos abiertos propietario (Arboleda \& Montoya, 2017). Los datos abiertos pueden ser subidos por entidades del estado o entidades descentralizadas o asociaciones que representan los intereses del sector privado que han sido previamente registradas.

\subsection{Estado actual de datos abiertos en salud en Colombia}

Se observa que las entidades del gobierno a nivel nacional (ministerios) ya han empezado a trabajar bajo los lineamientos de datos abiertos establecidos por el MINTIC. En el mapa de ruta del Plan Vive Digital (20142018) se priorizaron 6 proyectos orientados a la apertura de datos de diferentes entidades gubernamentales, dentro de los cuales se encuentra el proyecto de "Apertura de datos sobre prestación de servicios de salud, salud pública y gestión de riesgo en salud", liderado por el Ministerio de Salud y Protección Social (MSPS). La implementación del programa se realiza de manera progresiva. La iniciativa está orientada hacia un mapa de ruta de cuatro niveles: inicial, básico, avanzado y mejorado; estos van desde la puesta en funcionamiento de la infraestructura de forma básica, que actualmente se encuentra funcionando, hasta el uso de herramientas de la web semántica que permitan inteligencia de negocios en los datos y así lograr dar valor agregado a los datos publicados.

EI MSPS ha definido sus planes de datos abier-

14 https://catalogodatos.gub.uy/organization/ministerio-de-salud-publica

http://datos.gob.cl/organization/ministerio_de_salud

http://www.datosabiertos.gob.pe/secciones/da_datos_abiertos_directorio.asp?pag=9\&bloquePag=0 19 https://www.minsalud.gov. co/Paginas/datos-abiertos.aspx

https://www.minsalud.gov.co/Paginas/datos-abiertos.aspx

http://www.mintic.gov.co/

http://datosabiertoscolombia.cloudapp.net/frm/buscador/frmBuscador.aspx

https://socrata.com/

Pensamiento Americano Vol. 11 - No. 21 • Julio-Diciembre · Corporación Universitaria Americana • Barranquilla, Colombia • ISSN: 2027-2448 . http://coruniamericana.edu.co/publicaciones/ojs/index.php/pensamientoamericano 
tos para los periodos 2013-2015 y 2016-2017, con el objetivo de avanzar en las metas planteadas en la iniciativa de Gobierno Abierto (MSPS, 2017). Los principales logros destacados en estos planes es la construcción de 7 aplicaciones móviles para apoyo a la ciudadanía las cuales consumen información de los dataset disponibles, las cuales se han subido a las tiendas de Apple y Google Play: Autocuídate ${ }^{21}$, usada para actividades de autocuidado para control de embarazo basándose en: control del recién nacido, vacunas, agudeza visual, tamizaje neonatal, control de placa bacteriana, crecimiento y desarrollo y aplicación de vitamina k. (2014); SaluDatos ${ }^{22}$, para dar a conocer datos estadísticos de la salud en Colombia basándose en indicadores de salud, departamentos y municipios; ColombiaSiVigila, permite la consulta de clasificación de eventos como enfermedades transmisibles, enfermedades no transmisibles, enfermedades de causa externa, directorio de secretarias de salud, generalidades del evento, acciones de control, apoyo y diagnóstico diferencial; Discapp ${ }^{23}$, la cual presenta la oferta institucional y servicios de salud para personas con discapacidad; $\mathrm{GPCr}^{24}$, que es una guía de práctica clínica como herramienta de soporte a las decisiones médicas; ClicSalud ${ }^{25}$, la cual presenta datos con respecto a la calidad de salud de las EPS e IPS, el termómetro de precios de medicamentos.

Al consultar a través del portal de Gobierno en Línea, los datos ofrecidos por el MSPS ${ }^{26}$, se observa que existe un total de 127 conjuntos de datos publicados en diversas temáticas. Algunas de las temáticas presentadas en este portal y clasificadas de mayor relevancia son: Registro de Prestadores de Instituciones de Salud, Termómetro de Precios de Medicamen- tos, Medicamentos del POS, Procedimientos del POS, Número de afiliados por departamento, régimen y administradora para el año 2017, Calidad en Salud IPS, Calidad en Salud EPS, Indicadores de Salud y Protección Social, Información de riesgos y situaciones de interés salud pública, Servicios de salud para personas con discapacidad, entre otras.

Los datos con respecto a salud, también pueden ser publicados a nivel de los entes territoriales como alcaldías o secretarias departamentales o municipales. Si bien la estructura provista por el portal está disponible para que las alcaldías puedan presentar datos abiertos que muestren los resultados de su gestión, muy pocos municipios del país utilizan estas facilidades. Se observa que la Alcaldía de Medellín es una de las más activas en la presentación de datos abiertos que consolidan información relevante de salud como indicadores de Sistema de Vigilancia Epidemiológica (2008 a 2015), atención en procedimientos, hospitalización y consulta externa $(2015,2016)$, estado nutricional de la población menor de 6 años $(2015,2016)$, entre otros.

\subsection{Participación en la iniciativa de datos abiertos en Colombia}

La estrategia de Gobierno Línea, promueve desde su portal cinco formas de la manera como las entidades territoriales, las entidades descentralizadas, las universidades, los grupos de investigación y la comunidad en general, pueden aprovechar los datos abiertos como una estrategia para mejorar su gestión.

\section{Descubre}

Permite el acceso a conjuntos de datos pu- 
blicados por las entidades nacionales y territoriales. Es una funcionalidad disponible para todos los interesados (ciudadanos, empresas y entidades públicas y privadas) quienes pueden buscar conjuntos de datos por diferentes criterios (por categoría del dato, entidad que lo publica), visualizarlos de diferentes maneras y descargarlos en diferentes formatos.

\section{- Publica}

Esta es una funcionalidad disponible para que las entidades nacionales y territoriales publiquen los datos abiertos sobre los temas relacionados con su gestión. Para subir los datos deben estar previamente autorizados y pueden acceder a través del usuario y la contraseña.

\section{- Visualiza}

Permite la visualización de aplicaciones gráficas construidas a partir de datos existentes en el catálogo de datos abiertos.

\section{- Participa}

Los ciudadanos y demás entidades o empresas pueden solicitar datos abiertos que requieran o necesiten para sus análisis; esta solicitud debe ir acompañada de una justificación la cual se hace pública en el portal, asimismo la respuesta de los administradores del catálogo indicando si la solicitud fue aprobada o no. El hecho de que cualquier entidad o ciudadano pueda hacer una petición abierta sobre información que necesita de las entidades gubernamentales, busca generar una cultura de transparencia y acceso a la información, que rompe con el esquema tradicional de dificultad de acceder a la información del estado.

\section{- Herramientas}

A través de esta opción se ofrecen videos, guías y diversos materiales que orientan a la comunidad sobre el uso del portal. Dentro de las herramientas se encuentra una sección de interés para emprendedores, estudiantes y desarrolladores de aplicativos de software, puesto que da orientaciones técnicas acerca de la manera como se puede utilizar las librerías de desarrollo para acceder a los datos abiertos disponibles en la plataforma. Esta es una de las principales acciones que el MINTIC está impulsando y patrocinando a través de proyectos emprendedores, para fomentar el uso de los datos abiertos.

\section{Retos abiertos alrededor del enfoque de datos abiertos en salud}

Se reconoce el esfuerzo que Colombia está haciendo para posicionar los datos abiertos como una estrategia de buen gobierno y se identifican evidencias del trabajo que se ha iniciado en este sentido en el Ministerio de Salud y Protección Social. No obstante, se percibe que aún existen retos abiertos para buscar que el enfoque de datos abiertos en salud se convierta en una realidad.

\section{Cambio de cultura de los organismos res- ponsables de los datos para compartirlos.}

En muchas de las entidades territoriales o instituciones de salud, existe aún la cultura de que el poder se mantiene mientras se conserve el control sobre los datos. Este es quizás uno de los retos más importantes que deben ser trabajados desde las instituciones generadoras de datos. El objetivo es permitir a los ciudadanos el acceso a datos importantes disminuyendo derechos de acceso de forma que permita sacar de estos provecho y aumentar el indicador de percepción de satisfacción en acceso a los datos por parte de los ciudadanos e interesados y aumentando la publicación de datos bajo demanda (Kitsios \& Kamariotou, 2018) (Hossain, Dwivedi, \& Rana, 2016) (Sieber \& Johnson, 2015).

Una de las maneras de ir trabajando en el cambio de cultura, es la formación de los ciudadanos sobre la importancia en el acceso, 
uso y explotación de los datos abiertos como un recursos que no solo les permite tener acceso a la información, sino además que pueden hacer con los datos para informar y para sacar provecho económico de estos (Huijboom \& Broek, 2011) (Hossain et al., 2016).

\section{Empoderamiento de las entidades territo- riales que les permite entender y visualizar las potencialidades de este enfoque.}

El entendimiento de la importancia de los datos abiertos como herramienta de transparencia en gestión se convierte en un aspecto esencial para comunicar los indicadores alcanzados en programas y proyectos de índole social a ciudadanos y entidades públicas y privadas; en la medida en que las instituciones de salud se apropien la filosofía, finalidad, proceso de publicación, tecnología y comunicación con los interesados se podrá crecer regionalmente en este sentido. (Veljković, Bogdanović-Dinić, \& Stoimenov, 2014) (Zuiderwijk \& Janssen, 2014).

Desde la perspectiva de las alcaldías municipales, los datos abiertos pueden dar visibilidad a las gestiones del municipio y mostrar la transparencia de la gestión; asimismo las entidades descentralizadas que manejan temas sensibles a la comunidad pueden dar evidencia de su gestión efectiva a través de los datos abiertos.

\section{Hacer operativos los lineamientos de anoni- mización de los datos.}

En todo proceso de datos abiertos es necesario satisfacer las reglamentaciones en términos de privacidad de información controlando que la información publicada sea útil para la toma de decisiones y exista compatibilidad entre el derecho a la información y el derecho a la protección de los datos personales (Janssen, Charalabidis, \& Zuiderwijk, 2012) (Jarolímek \& Martinec, 2016).

A nivel internacional la norma ISO/IEC
29100:2011 define los roles y la responsabilidad de los actores involucrados en el ciclo de vida de los datos personales, permitiendo ser una referencia para la definición de una estrategia para protección de la privacidad, definiéndose aspectos como: controlador de datos, datos personales y flujo transfronterizo de datos personales, en el caso que la información se transmita de país a país (Acosta, 2017).

En Colombia ya existen reglamentaciones en ese sentido como la ley 1272 del 6 de Marzo de 2014 en la cual se define el derecho al acceso a la información pública nacional, exceptuando el acceso a la información por daño de derechos a personas naturales o jurídicas (Congreso de la República, 2014). Asimismo el DANE, a través de la Dirección de Regulación, Planeación, Estandarización y Normalización (DIRPEN), creo los lineamientos para la anonimización de microdatos, evitando la publicación de información sensible confidencial, a través de un proceso de anonimización, que permite recopilar información personas sin necesidad de saber su identidad; lo que garantiza que los datos sean usados solo con fines estadísticos y se garantice el principio de confidencialidad (DANE, 2014).

El Ministerio de Salud y Protección Social, mediante la Dirección de Epidemiología y Demografía, trazo los lineamientos para Anonimización de Datos del Sistema Nacional de Estudios y Encuestas Poblacionales en Salud, los cuales se rigen por la ley 1272 y sugieren el uso de métodos de aleatorización o perturbación y reducción o generalización como técnicas de anonimización y la aplicación de procedimientos y técnicas en concordancia con el Código Nacional de Buenas Prácticas para las Estadísticas Oficiales (CNBP) definidas por el DANE (MINSALUD, 2016).

EI MINTIC en la Guía de datos abiertos en Colombia establece que para publicar datos 
abiertos se deben adelantar acciones de anonimización eliminando información sensible evitando afectar personas naturales o jurídicas, adoptando las excepciones para publicación de la información mediante el establecimiento de información: información pública clasificada e información pública reservada (MINTIC, 2016).

Es importante que estos lineamientos de anonimización se traduzcan a guías prácticas que orienten a las entidades territoriales en sus ejercicios de publicación de datos abiertos. Por ejemplo, la Alcaldía de Bogotá aplica estas recomendaciones en su Guía sobre Información Pública y Excepciones a la Apertura de Datos, la cual es usada por todas las entidades públicas del Distrito para identificación de información pública que se considera Clasificada o Reservada (Alcaldía Mayor de Bogotá, 2016).

\section{Aprovechamiento de nuevas oportunidades de productos y servicios TIC alrededor de los datos abiertos.}

Para que los datos abiertos generen valor agregado que beneficie a la comunidad en general, es necesario seguir estimulado al sector de las TIC para el desarrollo y creación de nuevos productos y servicios, garantizándose un verdadero impacto de los datos abiertos gubernamentales en el país, de forma que se creen soluciones móviles y web que lleven los datos abiertos a los ciudadanos. El fomento de este tipo de acciones se puede realizar a través de jornadas de capacitación en desarrollo de aplicaciones de datos abiertos y competencias que permitan la apropiación y difusión de los datos abiertos como insumo para la creación de aplicaciones y servicios (Kitsios, Papachristos, \& Kamariotou, 2017) (Jarolímek \& Martinec, 2016).

\section{1 Hacia un marco de trabajo integrado de datos abiertos en salud}

En esta sección se esbozan algunas ideas de la manera como se pueden articular y potenciar los esfuerzos que realizan el MINTIC, el MSPS y los entes territoriales con el propósito de avanzar hacia la madurez del ofrecimiento de datos abiertos en salud, que incluya aspectos de promoción y prevención y se articule, a su vez, a las políticas de datos abiertos propuesta por la OMS.

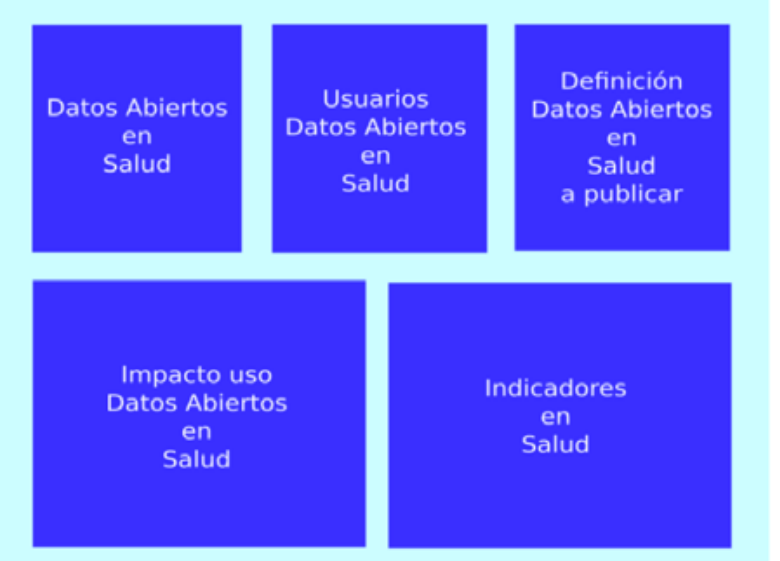

Figura 1. Marco Conceptual de Modelo de Datos en Salud. Adaptado de (Verhulst, Noveck, Caplan, Brown, \& Paz, 2014)

En primer lugar, es importante la definición de un marco general de trabajo para datos abiertos en salud. La figura 1 muestra una síntesis del marco conceptual de trabajo para datos abiertos en salud definido en Inglaterra (Verhulst, Noveck, Caplan, Brown, \& Paz, 2014) el cual puede servir de referencia para el MSPS en Colombia. Dicho marco identifica cinco elementos importantes: (a) los Datos Abiertos en salud, los cuales definen los tipos de formatos a usar y los aspectos legales para el uso de estos; (b) los Usuarios de Datos Abiertos, quienes serán clientes internos (el sistema de salud en Colombia con sus instituciones de salud y el personal de médico y observatorios de salud) y los clientes externos (pacientes, investigadores médicos, empresas y ciudadanos en general); (c) los Datos Abiertos en salud a publicar en donde se identifican los Datos abiertos del periodo pre patogénico (Prevención primaria: da- 
tos de Promoción general de la Salud, Prevención primaria: datos de Protección específica), Datos abiertos del Período patogénico (datos de diagnóstico precoz Tratamiento oportuno, datos de limitación del daño) y datos de Rehabilitación; (d) con respecto al impacto uso Datos Abiertos se evalúan los costos, la cantidad de usuarios de los datos abiertos, la mejora producida en la calidad de vida de los ciudadanos y en la prestación de los servicios por las entidades de salud; (e) en Indicadores se evalúa aspectos como el decrecimiento de enfermedades y de muertes, el aumento de medidas de prevención, el aumentos de adopción de prácticas saludables y el incremento en el número de pacientes atendidos.

Si bien la definición de Arquitectura de Datos Abierto para el sistema de salud representa un reto tecnológico importante, se considera que con la adopción de la plataforma de datos abiertos por parte del MINTIC, dicho reto ya se encuentra superado. La arquitectura propuesta permite que las diferentes entidades nacionales y regionales manejen sus propios repositorios o catálogos de datos abiertos, interoperables en una plataforma federada donde los datos están siendo gestionados y consumi- dos sin que las entidades responsables de su generación, pierdan el control sobre ellos (MINTIC, 2014).

La figura 2 presenta una visión de alto nivel del modelo operacional de datos abiertos en salud para Colombia, bajo responsabilidad del MSPS. Se trata de un gran catálogo formado por diferentes conjuntos de catálogos, que ofrecen datos de interés (dataset) para los diversos periodos (prepatogénicos, patogénicos, de rehabilitación), en los cuales se definen las diversas temáticas. En la figura se destacan los actores relevantes del ecosistema en sus tres categorías: Entidades Generadoras de Datos (EGD), Entidades Consumidoras de Datos (ECD) y Observatorios de Salud (OS). Dentro de las EGD se pueden mencionar las Secretarias de Salud Municipales y Departamentales, IPS / EPS, el Ministerio de la Protección Social y otras entidades del sector de la salud que participan de manera activa en el suministro y mantenimiento de los sistemas operativos de apoyo a sus funciones como el SIVIGILA, el RIPS, etc.

De lado opuesto aparecen las ECD como médicos, entidades de salud, pacientes, científicos, empresarios y ciudadanos en general por

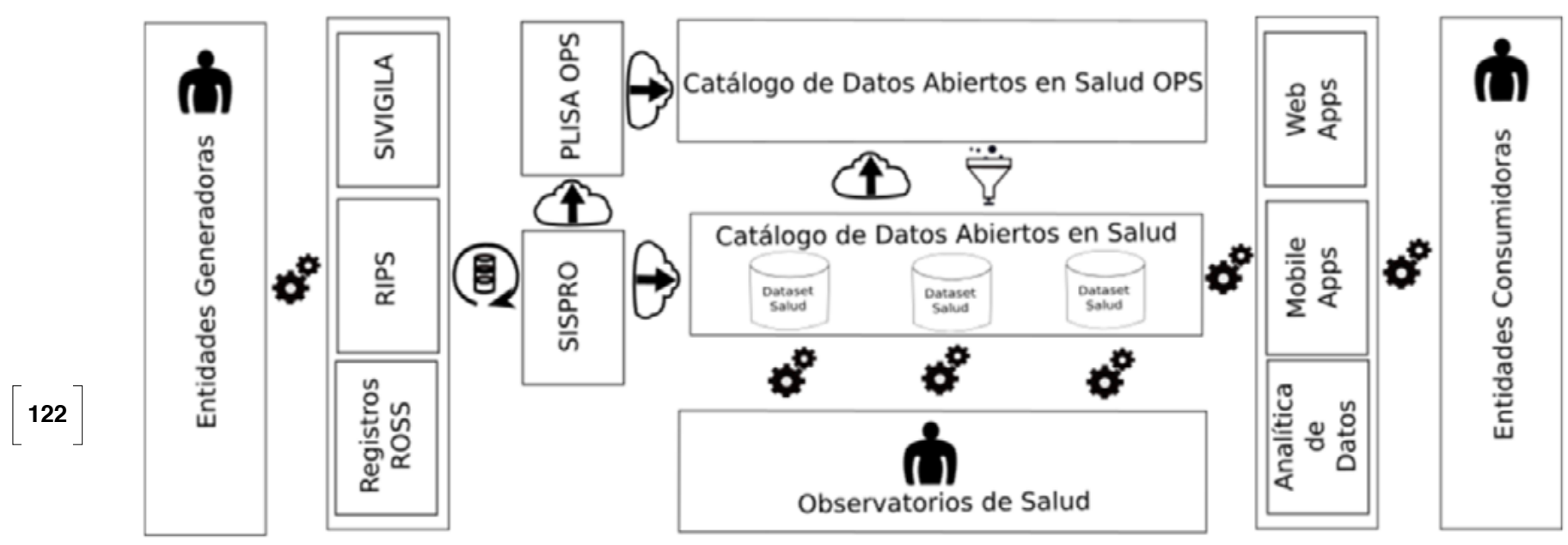

Figura 2. Propuesta del modelo operacional de datos abiertos de salud en Colombia

Pensamiento Americano Vol. 11 - No. 21 • Julio-Diciembre · Corporación Universitaria Americana • Barranquilla, Colombia · ISSN: $2027-2448$. http://coruniamericana.edu.co/publicaciones/ojs/index.php/pensamientoamericano 
medio del acceso abierto y descargue de los conjuntos de datos, a través de diversas soluciones informáticas como las aplicaciones móviles que ya han sido desarrolladas por el MSPS, aplicaciones web e incluso a través de herramientas especializadas en analítica de datos(MINSALUD, 2015).

Se destaca asimismo el papel de los observatorios de salud, como los actores orientados al seguimiento de la salud en Colombia y la orientación de políticas

que permite ir orientando los esfuerzos de salud hacia el tema de prevención, o si esta temática podría ser adoptada desde uno de los observatorios ya

establecido, como por ejemplo el de seguridad alimentaria y nutricional.

A partir de los sistemas transaccionales que soportan la gestión del sistema de salud integrado en SIVIGILA, existirán procesos claramente establecidos, principalmente automáticos, que realizan la extracción, consolidación y disposición de los datos abiertos en el Catalogo de Datos Abiertos en salud del Ministerio de Salud, de acuerdo a los lineamientos ya establecidos entre las diferentes entidades involucradas.

Con el propósito de establecer una articulación con el ecosistema de salud a nivel internacional, se hacen necesarios procesos de consolidación y filtro de información desde SISPRO hacia la Plataforma de Información en Salud de las Américas (PLISA) (OPS, 2017), y de manera complementaria desde el catálogo de datos abiertos de salud a nivel Colombia, hacia el catálogo de datos abiertos de salud manejado por la OMS.

El modelo operacional presentado en la figura 2, también intenta hacer una distinción entre los diferentes procesos de consolidación, trans- ferencia y cargue de datos que se realizan entre las soluciones informáticas y entre éstas los diversos actores ya mencionados:

: Se trata de soluciones informáticas responsables de la captura, validación, almacenamiento y gestión de datos que soportan los sistemas transaccionales de lado de las EGD, soluciones informáticas que toman como entrada los datos almacenados en los catálogos de datos abiertos para ofrecer soluciones puntuales de lado de las ECD o soluciones informáticas, como los sistemas de seguimiento, que soportan la gestión de los observatorios de salud.

Icono que representa los procesos consolidación de datos desde los sistemas transaccionales para ser dispuestos en los formatos, medios y niveles de acumulación definidos en los catálogos de datos abiertos.

: Proceso de adaptación de datos abiertos, solo de requieren los datos asociados a los indicadores los cuales son enviados al Catálogo de Datos Abiertos OPS.

(2)

: Proceso de integración de datos en el SISPRO, estos son datos provenientes de los Registros ROSS, RIPS y el SIVIGILA y su origen son las Entidades Generadoras.

\section{Conclusiones}

Se ha presentado en este trabajo una visión general del enfoque de datos abiertos como una estrategia potenciada por las TIC y utilizada por los gobiernos a nivel mundial para dar evidencia de transparencia y gobernabilidad de un país y se ha presentado una revisión general del estado de datos abiertos en el tema de la salud a nivel internacional, donde países como Estados Unidos y Reino Unido han tenido un papel de liderazgo y a nivel nacional donde si bien se reconoce el esfuerzo que el MINTIC y 
el MSPS han realizado en la estrategia de datos abiertos en salud, se observa que los datos publicados no tienen el nivel de representatividad deseable, lo que permite deducir que aún no se encuentran maduros los lineamientos y procedimientos que permiten la publicación de dichos datos por parte de las entidades responsables.

Este trabajo ha identificado retos actuales que deben ser enfrentados para seguir madurando en la aplicación del enfoque de datos abiertos en el tema de la salud. Dichos retos, son más de tipo cultural que tecnológico, buscando potenciar a las entidades territoriales para que tomen un papel activo en la publicación de sus datos, de manera especial, aquellos datos relacionados con la promoción de la salud.

A manera de propuesta, se esboza un marco conceptual de trabajo que puede servir de referente con mirar a contar con un catálogo de datos abiertos en Salud exclusivo, que permita que los entes territoriales avancen en el ofrecimiento de datos abiertos en salud de forma más integrada, articulándose a las políticas de datos abiertos propuesta por la OMS, MINTIC y MSPS. Asimismo la propuesta operacional que se esboza en este trabajo ilustra la manera como los diferentes actores y sistemas de información se articulan para crear un ecosistema que permita hacer mejor uso de los datos en salud y genere un mayor impacto al integrar datos de diversas fuentes.

Un adecuado manejo del catálogo de datos abiertos en el tema de la salud, contribuye a mejorar la transparencia del sistema de salud del país y a promover un trabajo articulado Universidad-Empresa-Estado, gracias a tener la información sincronizada que permita crear proyectos, planes y programas que se adapten a la realidad del sector salud a nivel regional y nacional. También aparecen posibilidades de desarrollo de aplicaciones e infraestructuras tecnológicas para los desarrolladores de productos tecnológicos permitiéndose igualmente un crecimiento de la industria TIC del país dedicada al sector de la salud. 


\section{Referencias}

Acosta, D. (2017). ISO / IEC 29100 : 2011 Una Introducción al Marco de Trabajo de Privacidad para la Protección de Información de Identificación Personal. SIC, 114-120.

Alcaldía Mayor de Bogotá. (2016). Guía sobre Información Pública y Excepciones a la Apertura de Datos (Vol. 14). Bogotá, Colombia.

Bonina, C. (2015). Cocreación, innovación y datos abiertos en ciudades de América Latina: lecciones de Buenos Aires, Ciudad de México y Montevideo.

CDC. (2018). Chronic Disease Indicators Data. Retrieved July 23, 2018, from https://chronicdata.cdc.gov/health-area/chronic-disease-indicators

CDPH. (2018). California Department of Public Health. Retrieved July 23, 2018, from https://www.cdph.ca. gov/Pages/CDPHHome.aspx\#

Congreso de la República. (2014). Ley de Transparencia y del Derecho a la Información Pública Nacional. 6 de Marzo de 2014. Bogotá, Colombia. Retrieved from http://wsp.presidencia.gov.co/Normativa/Leyes/Documents/LEY 1712 DEL 06 DE MARZO DE 2014.pdf

DANE. (2014). Lineamientos para la Anonimización de Microdatos. Bogotá, Colombia. Retrieved from http://www.dane.gov.co/files/sen/lineamientos/ DSO_020_LIN_08.pdf

DATA.GOV. (2018). The home of the U.S. Government's Open Data. Retrieved July 23, 2018, from http:// www.data.gov

DATA.NJ.GOV. (2018). Healthy New Jersey 2020. Retrieved July 23, 2018, from https://data.nj.gov/Health/Healthy-New-Jersey-2020/79id- pfue?category=Health\&view_name=Healthy-New-Jersey-2020

Eibl, G. (2013). Framework for Open Government Data platforms. Viena.

Elena, S. (2015). Datos abiertos para una justicia abierta: un análisis de caso de los Poderes de Brasil, Costa Rica y Perú.

Hossain, M. A., Dwivedi, Y. K., \& Rana, N. P. (2016). State-of-the-art in open data research: Insights from existing literature and a research agenda. Journal of Organizational Computing and Electronic Commerce, 26(1-2), 14-40. https://doi.org/10.1080/1091939 2.2015.1124007

Huijboom, N., \& Broek, T. Van Den. (2011). Open data: an international comparison of strategies. European Journal of ePractice, 12 (March/ April 2011), 1-13. https://doi.org/1988-625X

Janssen, M., Charalabidis, Y., \& Zuiderwijk, A. (2012). Benefits, Adoption Barriers and Myths of Open Data and Open Government. Information Systems Management, 258-268.

Jarolímek, J., \& Martinec, R. (2016). Analysis of Open Data Availability in Czech Republic Agrarian Sector. Agris on-Line Paper in Economics and Informatics, 8(3), 57-67. https://doi.org/10.7160/aol.2016.080306.Introduction

Khelladi, Y. (2015). Datos abiertos en educación , primeros alcances y lecciones.

Kitsios, F., \& Kamariotou, M. (2018). Open Data and High-Tech Startups Towards Nascent Entrepreneurship Strategies. Encyclopedia of Information Science and Technology, Fourth Edition, (November 2017), 3032-3041. https://doi.org/10.4018/978-1-5225-22553.ch 265

Kitsios, F., Papachristos, N., \& Kamariotou, M. (2017). Business models for open data ecosystem: Challenges and motivations for entrepreneurship and innovation. Proceedings - 2017 IEEE 19th Conference on Business Informatics, CBI 2017, 1(July), 398- 407. https://doi.org/10.1109/CBI.2017.51

Malavera Pineda, S. M. (2013). La Efectividad de las Empresas de Base Social en la Ciudad de Medellín. Ad-Gnosis, 2(2), 87-93.

MINSALUD. (2015). Guía Metodológica para Registros, Observatorios, Sistemas de Seguimiento y Salas Situacionales Nacionales en Salud de Colombia. Bogota. Retrieved from https://www.minsalud.gov.co/ sites/rid/Lists/BibliotecaDigital/RIDE/VS/ED/GCFI/ ROSS_0 01.11.2013.pdf

MINSALUD. (2016). Lineamientos para Anonimización de Datos del Sistema Nacional de Estudios y Encuestas Poblacionales en Salud. Bogotá, Colombia.

MINTIC. (2014). Lineamientos para la Implementación de Datos Abiertos en Colombia. Bogotá, Colombia.

MINTIC. Guía de datos abiertos en Colombia (2016). Colombia.

MSPS. (2017). Plan de Datos Abiertos 2016-2017 (Vol. 2). Bogotá.

National Prevention Council. (2011). National Prevention Strategy, (June).

NCCDPHP. (2018). All Chronic Surveillance Systems. 
Retrieved July 23, 2018, from https://www.cdc.gov/ chronicdisease/stats/

Noveck, B. S. (2014). The Open Data Era in Health and Social Care.

ODC. (2018). The Open Data Charter. Retrieved July 27, 2018, from https://opendatacharter.net/

OPEN.CANADA.CA. (2018). Canadian Chronic Disease Surveillance System 2000/2001- 2013/2014. Retrieved July 23, 2018, from https://open.canada.ca/ data/en/dataset/9525c8c0-554a-461b-a763-f1657acb9c9d

OPS. (2017). PLISA Plataforma de Información en Salud de las Américas.

Pane, J., Ojeda, V., \& Valdez, N. (2015). Dengue Open Data.

Rivillas, J. C., Ivo, J., Caicedo, M., Milena, C., Segura, C., \& Lucía, M. (2013). Registros, observatorios y sistemas de seguimiento en salud en colombia. Ministerio de Salud, 7.

Sieber, R. E., \& Johnson, P. A. (2015). Civic open data at a crossroads: Dominant models and current challenges. Government Information Quarterly, 32(3), 308-315. https://doi.org/10.1016/j.giq.2015.05.003

SOCRATA. (2018). Socrata Case Studies. Retrieved July 23, 2018, from https://socrata.com/case-studies/

Veljković, N., Bogdanović-Dinić, S., \& Stoimenov, L. (2014). Benchmarking open government: An open data perspective. Government Information Quarterly, 31(2), 278-290. https://doi.org/10.1016/j.giq.2013.10.011

Suárez, D. (2011). Influencia de los sistemas de la información en las organizaciones. Pensamiento Americano, 4(6), 31-34.

Verhulst, S., Noveck, B. S., Caplan, R., Brown, K., \& Paz, C. (2014). The Open Data Era in Health and Social Care. Londres.

Zuiderwijk, A., \& Janssen, M. (2014). Open data policies, their implementation and impact: A framework for comparison. Government Information Quarterly, 31(1), 17-29. https://doi.org/10.1016/j.giq.2013.04.003 